\title{
Periodic exophthalmos associated with the blue rubber bleb naevus syndrome: a case report
}

\author{
I. G. RENNIE, ${ }^{1}$ J. R. SHORTLAND, ${ }^{2}$ J. M. MAHOOD,$^{3}$ AND B. H. BROWNE \\ From the Departments of ${ }^{1}$ Ophthalmology, ${ }^{2}$ Pathology, and ${ }^{3}$ Dermatology, University of Sheffield
}

SUMMARY A 71-year-old woman with the blue rubber bleb naevus syndrome experienced 4 episodes of self-limiting unilateral exophthalmos. It is suggested that a vascular malformation in the orbit is responsible for this phenomenon. A biopsy sample was taken of one of the cutaneous lesions and studied by light and transmission electron microscopy. The histological appearances are discussed in relation to possible mechanisms causing this periodic exophthalmos.

In 1958 Bean' gave the name 'blue rubber bleb naevus syndrome' to a condition characterised by erectile, bluish naevi of the skin associated with angiomatosis of the gastrointestinal tract. Since this classic description was published several reports have established that other systems may also have such vascular malformations. The lesions have been described in the nasopharynx ${ }^{2}$ liver, ${ }^{34}$ lung, ${ }^{3}$ pleura, ${ }^{35}$ heart, $^{5}$ and central nervous system. ${ }^{5}$ Recently a case of ocular involvement has been described, in which lesions were present in the conjunctiva, iris, and retina. ${ }^{6}$

We report a case of blue rubber bleb naevus syndrome associated with periodic exophthalmos, comment on this phenomenon, and describe the ultrastructure of one of the cutaenous lesions.

\section{Case report}

The patient, a 71-year-old female, presented to the Ophthalmology Department as an emergency with a history of having awakened that morning with a painful left eye. She had also noted that the vision in that eye was impaired.

On examination a marked degree of proptosis was present on the left side. This was measured with a Hertel exophthalmometer; the difference between the 2 sides was $9 \mathrm{~mm}$ (right $7 \mathrm{~mm}$, left $16 \mathrm{~mm}$ ). A complete ptosis was present on the left side. The lids could be opened only passively, with extreme difficulty, over the tense globe to reveal a chemotic conjunctiva. The eye movements on that side were grossly restricted. The corrected visual acuity was $6 / 9$

Correspondence to Dr I. G. Rennie, Hallamshire Hospital, Glossop Road. Sheffield S10 2JF. in the right eye. An accurate visual acuity was unobtainable in the left eye owing to the ptosis and distressed state of the patient. The pupils were equal in size; however, an afferent pupil defect was present on the left side. Funduscopy revealed some congestion of the left retinal veins. Several lesions on the skin of face and neck were noted. The largest, present on the lobule of her right ear, measured 0.5 $\mathrm{cm}$ in diameter (Fig. 1). The lesions consisted of discrete, dark blue, easily compressible swellings

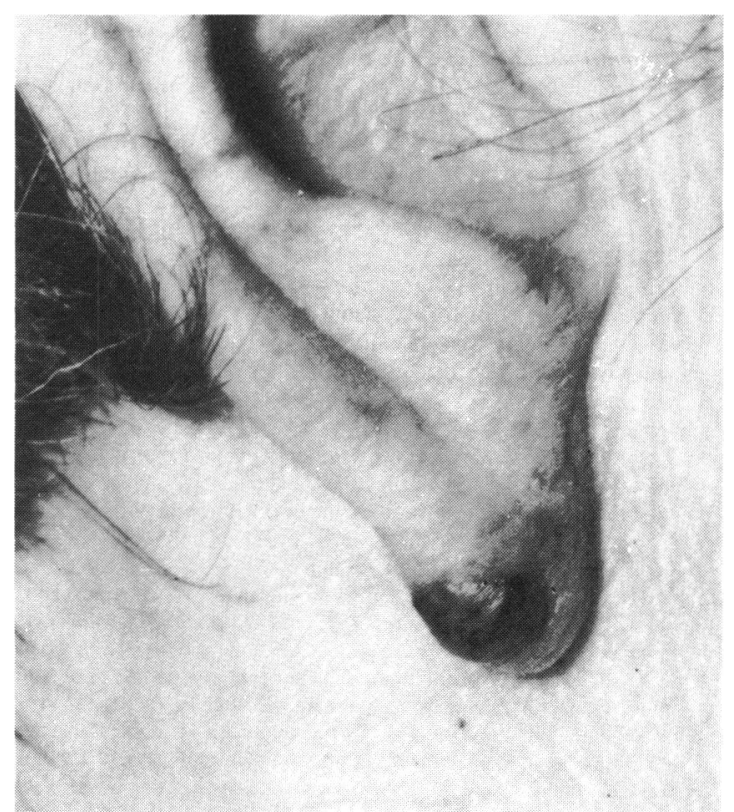

Fig. 1 Blue rubber bleb naevus present on the lobule of right ear. 


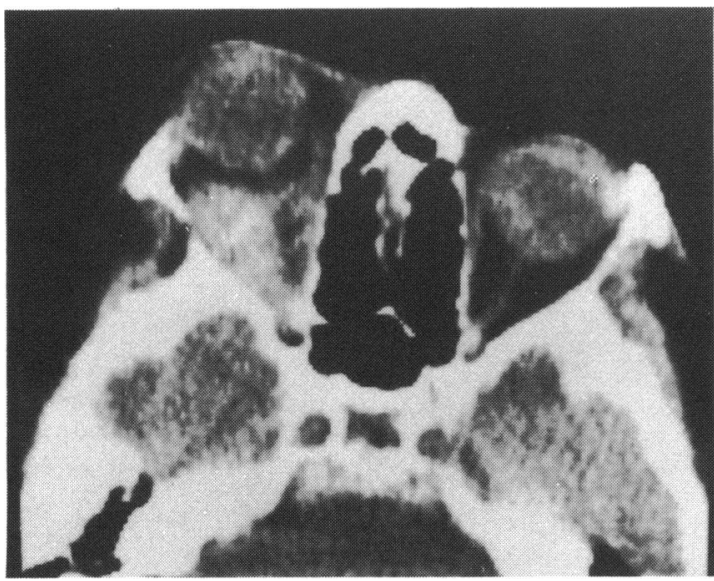

Fig. 2 Computerised axial tomography of the orbits showing a high-density lesion in the left orbit separated from the globe by a narrow rim of fat. Note the marked degree of proptosis of the left eye.

which had a bladder-like consistency. After the lesions were emptied on digital pressure they were seen to refill completely in a few seconds. Multiple haemangiomas were also present on the tongue and a few over the surface of the buccal mucosa. The patient stated that, although these lesions had been present for many years, they were not present in childhood. A large lesion on her lower lip bled profusely on one occasion, but had been treated successfully by radiotherapy 8 years earlier. Apart from this episode of bleeding there was no history of gastrointestinal haemorrhage.

Despite the absence of any subconjunctival haemorrhage, bleeding from or into an orbital vascular malformation was considered as the probable cause

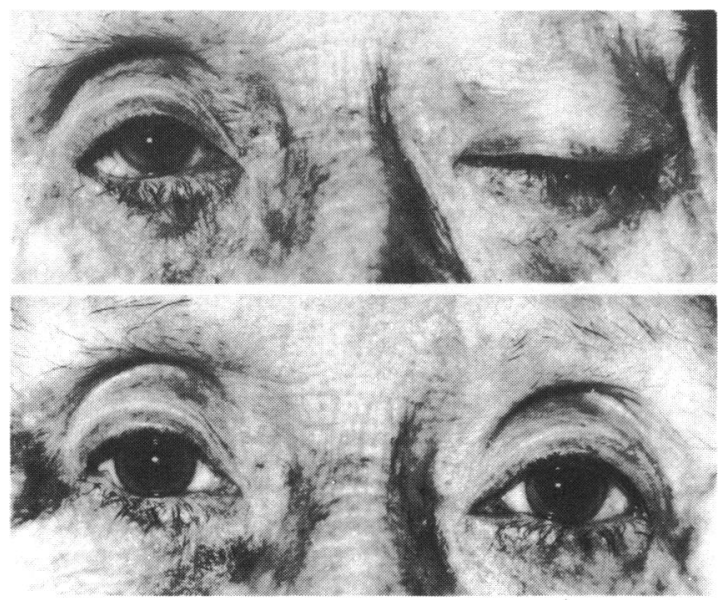

Fig. 3 The appearance of the eyes: (upper) during an acute episode of exophthalmos; (lower) two days later.

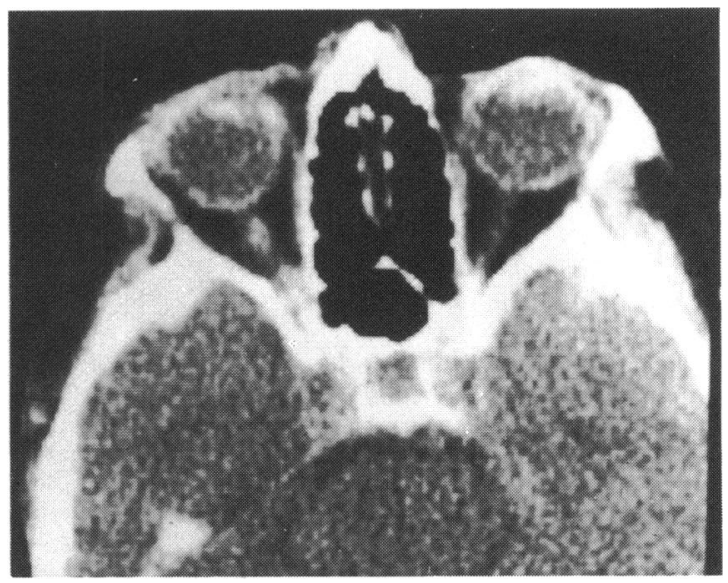

Fig. 4 Computerised axial tomography of the orbits (with contrast enhancement) after the resolution of the second episode of proptosis. A small high-density lesion is present close to the left optic nerve.

of the sudden proptosis. The patient was admitted for investigation. Computerised axial tomography of the orbits was performed. This revealed a high-density intraconal lesion, separated from the posterior surface of the globe by a narrow rim of fat (Fig. 2). At $2000 \mathrm{~h}$, some 4 hours after the initial examination, the patient was re-examined. The ptosis was now incomplete and slight horizontal eye movements were now possible. In addition the patient noted that the pain was now less severe. In view of the improving physical signs a conservative approach was adopted, and the patient was treated with analgesics. The following morning both proptosis and ptosis had almost completely disappeared. Measurement of the proptosis revealed a clinically insignificant $2 \mathrm{~mm}$ disparity between the 2 sides. Slight diplopia was noted on the extremes of gaze, otherwise the eye movements had returned to normal. The corrected visual acuity in the left eye was now 6/9 part.

At $0400 \mathrm{~h}$ on the fourth day of admission the patient was awakened by a recurrence of the severe pain in her left eye. Examination revealed the same clinical picture seen on admission, the left eye was proptosed (the disparity measuring $8 \mathrm{~mm}$ ), with a complete ptosis and external exophthalmoplegia (Fig. 3, upper). The patient was again treated only with analgesics. Computerised axial tomography of the orbits, this time with contrast enhancement, was performed later that day. Again the same high density lesion was present in the left orbit. The proptosis resolved over the next 48 hours (Fig. 3, lower).

On the seventh day after admission computerised axial tomography with enhancement was repeated; it revealed a small residual lesion in close relation to the optic nerve (Fig. 4). 
Fig. 5 Light photomicrograph of the cutaneous lesion showing a benign haemangioma, the walls of which contain bundles of smooth muscle in some areas. ( $H$ and $E$, $\times(1 \cdot 7)$.

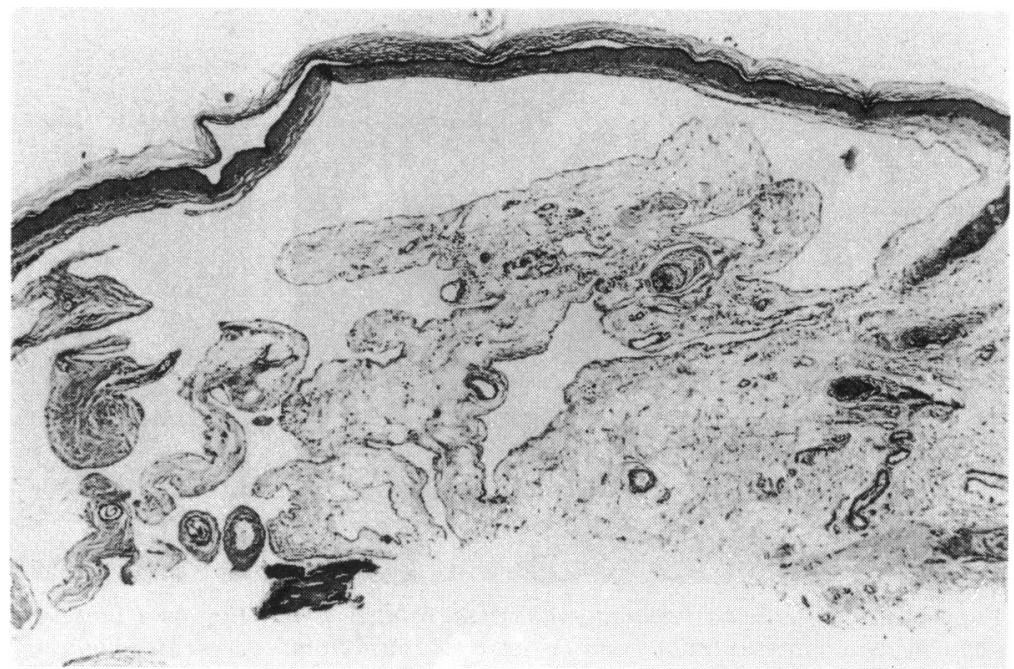

Further investigations, including full blood count, orbital venograms, and carotid angiography, were normal. Attempts to produce the proptosis by bending or straining were unsuccessful. The lesion on the right ear was biopsied under local anaesthesia. The patient remained asymptomatic and was discharged 12 days after admission.

department with a similar episode of painful proptosis of the left eye. Again the proptosis had been present on waking. The ocular findings were similar to the first admission, with a proptosed $(8 \mathrm{~mm}$ difference between the 2 sides), and a chemotic eye. As in the previous admission a complete ptosis was present as well as a gross restriction in the eye Eight weeks later the patient returned to the movements. The corrected visual acuity in the

Figs $6 \mathrm{a}$ and $6 \mathrm{~b}$ Electron micrographs of 2 areas from the same cavernous channel. $(\times 3636)$

Fig. 6a Smooth muscle cells lie immediately subjacent to the endothelium.

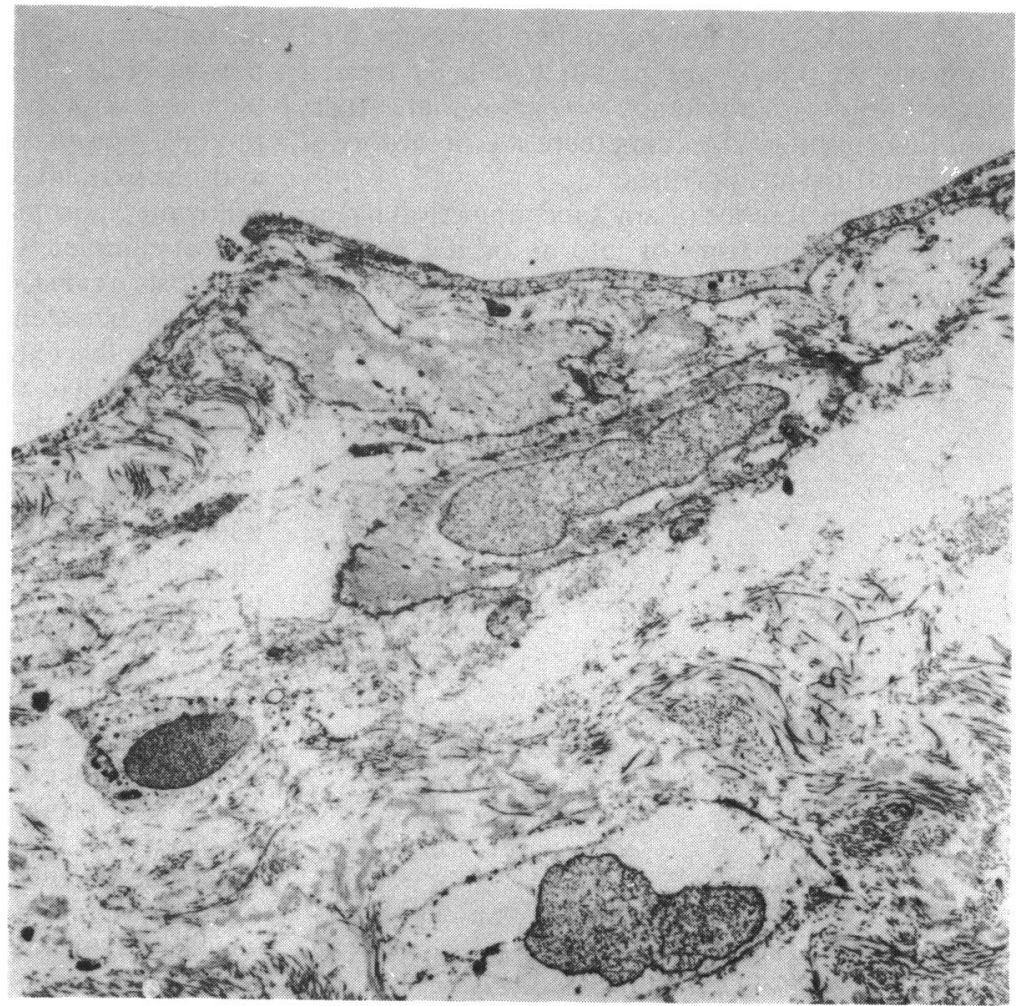


affected eye was $6 / 12$. On this occasion an afferent pupil defect was not noted. The patient was given sedatives and analgesics. The next morning the proptosis had resolved. She was discharged the following day, only to return 3 days later with similar symptoms. As on all the other occasions the proptosis had been present on waking. A similar regimen of sedation and analgesics was employed, with the now predictable resolution of symptoms by the following day. The patient was discharged 2 days later.

\section{HISTOLOGICAL STUDIES}

The cutaneous specimen was fixed in $10 \%$ formol saline for 24 hours and subsequently processed through graded alcohols to paraffin wax. Sections were cut at $4 \mu \mathrm{m}$ and stained with haematoxylin and eosin, elastic Van Gieson, acid red direct yellow, and Martius scarlet blue for standard light microscopy.

Electron microscopy specimens were obtained from the paraffin block, and after removal of the wax, postfixed with osmium tetroxide and again dehydrated and embedded in epoxy resin. Sections were cut on an LKB ultratome at $0.5 \mu \mathrm{m}$, stained with toluidine blue, and examined with the light microscope for selection of appropriate areas. Subsequent ultrathin sections were cut at $80 \mathrm{~nm}$, stained with lead citrate and uranyl acetate, and examined with an AEI Corinth electron microscope with an accelerating voltage of $60 \mathrm{Kv}$ and magnifications ranging from 1200 to 20000 diameters.

Light microscopy. The lesion from the ear lobule had the configuration of a benign cavernous haemangioma. The endothelial cells were low and attenuated. The stromal septa between the blood spaces varied; some comprised connective tissue, while others contained substantial smooth muscle components (Fig. 5).

Electron microscopy. The blood spaces were lined by a low pavemented endothelium, showing no exceptional features. The adjacent stroma reflected the picture seen on light microscopy. Fibroblasts and collagen bundles were the sole feature in some areas of the wall, while in other areas of the same blood space recognisable bundles of smooth muscle cells were present (Figs. 6a,b). Ultrastructurally these cells were more plump than the fibroblasts and contained orderly filaments related to dense bodies, both in the cytoplasm and at the plasma membrane. Abundant micropinocytotic vesicles were present, and a thin investing basement membrane was discernible. Collagen bundles were present adjacent to the smooth muscle cells lying immediately subjacent to the endothelium.

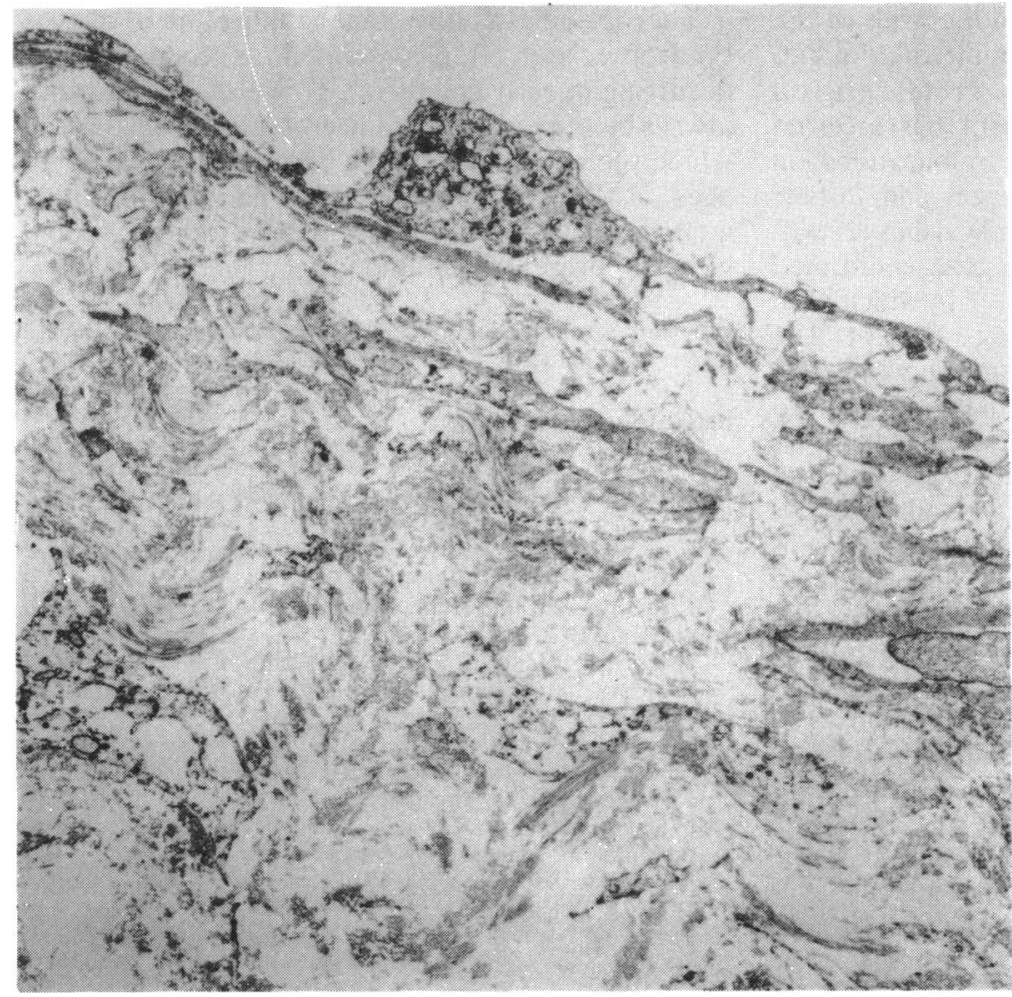

Fig. 6b Fibroblasts and collagen constitute the vascular stroma. 


\section{Discussion}

Intermittent proptosis is a rare but well recognised clinical entity. Typically the clinical picture is that of a transient, unilateral proptosis of variable duration lasting from a few seconds to a few days, which may be precipitated by manoeuvres which increase the orbital venous pressure. The commonest cause of this condition is an orbital varix. ${ }^{7}$ This may be a primary congenital venous malformation or secondary to an arteriovenous shunt. ${ }^{89}$ The clinical picture presented by the patient in this report differs from the classical description of intermittent proptosis. A fundamental difference is the inability to produce the proptosis by increasing the orbital venous pressure. Furthermore normal orbital angiography and venography would suggest that the lesion is unlikely to be an orbital varix. We feel that the most likely cause for her periodic exophthalmos is the presence of an expansile vascular malformation in her orbit similar in nature to her cutaneous haemangiomas.

The lesion removed from the ear lobule in our patient was a benign cavernous haemangioma. The endothelial cells were attenuated throughout, unlike the cuboidal layer reported by Fine et al. ${ }^{10}$ and claimed by those authors to be characteristic of the blue rubber bleb lesions. Rice and Fisher ${ }^{3}$ reported that the dilated channels constituted an endothelial layer only, unlike our case, in which the walls of the blood spaces were composed of fibromuscular tissue, a feature emphasised by Bean ${ }^{1}$ in his original description. The stromal component has received considerable attention, and from publications on these lesions 3 types emerge. Fretzin and Potter ${ }^{2}$ observed fibrous walls, whereas Walshe et al. ${ }^{11}$ observed muscular elements. Our case conformed most to that described by Fine et al. ${ }^{10}$ in which both muscular and fibrous components are present. The ultrastructural evidence that we found shows clearly that smooth muscle cells and fibroblasts are applied closely to the endothelial spaces. They thus constitute a specific part of the vascular wall rather than an adjacent stromal component incorporated passively. It is interesting to note that both smooth muscle cells and fibroblasts are in evidence within the wall of the same vascular channel. A similar observation was made in a rather more complex lesion involving the brain. ${ }^{12}$ As a result of their studies Rosenblum et al. ${ }^{12}$ suggest that the blue rubber bleb lesion may encompass a wide variety of vascular malformation ranging from capillary telangiectasis to arteriovenous malformations.

It has been recognised previously that the cutaneous lesions of the blue rubber bleb naevus syndrome are capable of episodic swelling. Indeed our patient described an intermittent swelling of the lesions on her tongue and buccal mucosa. Episodic pain with or without an increase in size is also a feature of these lesions. ${ }^{1013}$ It is of interest to note that the pain associated with these lesions occurs typically at night. The exact mechanism for the variation in size of the lesions and the production of pain is unknown. Fine et al. ${ }^{10}$ suggested that the pain is probably caused by contraction of the smooth muscle elements contained in the lesions.

Our studies on the ultrastructure of the cutaneous lesions confirm the presence of smooth muscle cells, closely related to the endothelial lining of the vascular channels. It is possible that segmental contraction of these smooth muscle elements in the distal aspect of the malformation could lead to dilatation of the proximal vascular channels and an increase in size of the lesion, and this mechanism may be responsible for at least the initial increase in size of the lesion. Once fully expanded, the orbital congestion produced would lead to local ischaemia and subsequent relaxation of the smooth muscle elements. An alternative explanation is that the vascular malformation is acting as ball valve, blocking the venous return from the orbit. Rowbottom and Little ${ }^{14}$ described a case of haemangioma causing intermittent proptosis by compressing the venous drainage from the orbit at the superior orbital fissure. It is possible that both mechanisms may exist together.

The lesions of the blue rubber bleb naevus syndrome are often described as congenital or occurring in early childhood. However, this may not always be the case. Sakurane et al. ${ }^{13}$ describe a case in which the cutaneous lesions developed between the ages of 19 and 23 years. Our patient was quite adamant that her lesions developed in adulthood and were not present in childhood.

We thank Mr A. J. Dark for allowing the publication of his clinical material and $\mathrm{Mr} \mathrm{T}$. Gray for his technical assistance.

\section{References}

1 Bean WB. Blue rubber bleb naevi of the skin and gastrointestinal tract. Vascular Spiders and Related Lesions of the Skin. Springfield: Thomas, 1958: 178-85

2 Fretzin DF. Potter B. Blue rubber bleb nevus. Arch Intern Med 1965; 116: 924-9.

3 Rice JS. Fisher DS. Blue rubber-bleb nevus syndrome. Arch Dermatol 1962; 86: 163-71.

4 Hoffman T. Chasko S. Safai B. Association of blue rubber nevus syndrome with chronic lymphocytic leukemia and hypernephroma. Johns Hopkins Med J 1978; 142: 91-4.

5 Waybright EA. Selhorst JB, Rosenblum WI, Sater CG. Blue rubber nevus syndrome with CNS involvement and thrombosis of a vein of Galen malformation. Ann Neurol 1978; 3: 464-7.

6 Crompton JL. Taylor D. Ocular lesions in the blue rubber naevus syndrome. Br J Ophthalmol 1981; 65: 133-7.

7 Duke-Elder S, ed. System of Ophthalmology. London: Kimpton. 1974: 13 (2): 825-34.

8 Walsh FB, Dandy WE. Pathogenesis of intermittent exophthalmos. Arch Ophthalmol 1944; 32: 1-10. 
9 Lloyd GAS. Wright JE. Morgan G. Venous malformation in the orbit. Br J Ophthalmol 1971: 55: 505-16.

10 Fine RM. Derbes VJ. Clark WH. Blue rubber bleb nevus. Arch Ophthalmol 1961: 84: 802-5.

11 Walshe MM. Evans CD. Warin RP. Blue rubber bleb nevus svndrome. Br Med J 1966: ii: 931-2.

12 Rosenblum WI. Nakoneczna I. Konerding FS. Wochlin P. Ghata NR. Multiple vascular malformations in the blue rubber bleb naevus' syndrome: a case with aneurysm of vein of Galen and vascular lesions suggesting a link to the Weber-Osler-Rendu svndrome. Histopathologv 1978: 2: 301-11.

13 Sakurane HJF. Sugai T. Suito T. The association of blue rubber bleb nevus and Maffucci's svndrome. Arch Dermatol 1967: 95: 28-36.

14 Rowbottom GF. Little E. Haemangioma of the orbit. Br J Ophthalmol 1966: 50: 47-9. 